\title{
EFFECT OF NUCLEATION ON THE STABILITY OF BCC METALS
}

\author{
A. VERMa, M.L. VERMA \\ Department of Physics, G.G.D.S.D. College, Palwal-121102, India \\ AND R.P.S. RATHORE \\ Department of Physics, R.B.S. College, Agra-282002, India
}

(Received October 18, 1996)

\begin{abstract}
Numerical calculations are made of theoretical strength and range of stability of a perfect uniaxially stressed crystal lattice of bcc vanadium, niobium and tantalum in the framework of extended generalised exponential potential by applying Born stability criteria. Two ranges of stability, a bcc phase and a body centered tetragonal phase are found to exist. The computed values of theoretical strength and strain of bcc $\mathrm{V}, \mathrm{Nb}$ and $\mathrm{Ta}$ agree reasonably well with the experimental limits.
\end{abstract}

PACS numbers: $62.20 . \mathrm{Dc}$

\section{Introduction}

The theoretical or ideal strength of a perfect crystal is defined as the strength at which a perfect lattice becomes mechanically unstable [1]. Since a perfect crystal is homogeneous throughout, it cannot become mechanically unstable till the system lowers its total energy by spontaneously undergoing uniform deformations. These deformations called twinning or nucleation [2] leading to structural transition from tetragonal to orthorhombic phase have direct bearing on the process of hot superconductors. The present study, however, deals with the problem of theoretical strength and stability of bcc $\mathrm{V}, \mathrm{Nb}$ and $\mathrm{Ta}$ under nucleation from cubic to tetragonal phase. In fact, the theoretical strength represents an upper bound or limit to the actual strength of the crystalline solids, therefore its calculation is a problem of central importance and assumes interesting dimensions because it is thought that the strength of some metallic whiskers or fine filaments approach the theoretical limit. Before the development of the high-strength crystalline whiskers, this problem was of prime importance since the calculated values of tensile strength [3-6] were typically greater than the experimental ones by a factor of 100 or more. 
Currently, this problem is still of theoretical as well as practical interest because there now exists means for obtaining high-strength crystalline whiskers which are evidently relatively free from microscopic defects (i.e. devoid of impurities and lattice imperfections). In addition, the problem of calculating the theoretical strength of perfect crystals is relevant to our understanding of many phenomena occurring in solid state physics such as:

(i) the nature of a material whether brittle or ductile $[4,7]$;

(ii) the definition of dislocation core radii [8,9];

(iii) the loss of coherency occurring at particle matrix interfaces $[10,11]$ are the problems which involve the ideal strength of solids. Many workers [12-16] have studied this problem of theoretical strength both for undeformed and deformed crystal lattices with various modes of deformations and with various forms of interactions between the atoms. Recently, Singh [17] has computed theoretical strength of noble metals in [100] direction by using a pseudopotential approach and has made a rigorous estimation of binding energy. Probably, the present knowledge of interatomic interactions in solids is still insufficient and an accurate quantitative calculation of the theoretical strength for most of the solids is hardly tractable.

The purpose of present communication is to apply Born stability criteria [1] using the extended generalised exponential potential (EGEP) to the problem involving any kind of lattice deformation, that is, either homogeneous expansion or contraction and interpret the results of detailed numerical computations carried out for bcc vanadium, niobium and tantalum. The present potential [18] has earlier explained not only the elastic and dynamical behaviour of bcc $\mathrm{V}$ and $\mathrm{Nb}$ but also of fcc Al recently. The computed values of theoretical strength and strain for bcc $\mathrm{V}, \mathrm{Nb}$ and $\mathrm{Ta}$ agree reasonably well with the experimental limits.

\section{Theory}

\subsection{Extended generalised exponential potential}

The attractive as well as the repulsive components of the generalised exponential potential [19] have been extended for representing their true and realistic nature. Extended generalised form of exponential potential coupling the $i$-th and $j$-th atoms separated by a distance $r_{i j}$ assumes the form

$$
\Phi_{m}\left(r_{i j}\right)=[D /(m-1)]\left[\mathrm{e}^{-m \alpha\left(r_{i j}-r_{0}\right)} /\left(\alpha r_{i j}\right)^{n}-m\left(\alpha r_{i j}\right)^{n} \mathrm{e}^{-\alpha\left(r_{i j}-r_{0}\right)}\right] .
$$

The average interaction (cohesive energy) energy per atom within the framework of EGEP is

$$
\Phi_{m}(r)=[D / 2(m-1)] \sum_{j}\left[\mathrm{e}^{-m \alpha\left(r_{j}-r_{0}\right)} /\left(\alpha r_{j}\right)^{n}-m\left(\alpha r_{j}\right)^{n} \mathrm{e}^{-\alpha\left(r_{j}-r_{0}\right)}\right],
$$

where $m$ and $n$ are the parameters which take care of electronic exchange and correlation effects and the three-body forces such as volume forces in an alternative and simpler form respectively, $D$ is the dissociation energy, $\alpha$ - the hardness parameter, $r_{0}$ - the equilibrium separation parameter and $r_{j}$ is the distance of the $j$-th atom from the origin given by

$$
r_{j}=\left(l_{1}^{2}+l_{2}^{2}+l_{3}^{2}\right)^{1 / 2} a_{0}=L_{j} a_{0}
$$


where $l_{1}, l_{2}$, and $l_{3}$ (either all even or all odd for a bcc lattice) are the integers of the position coordinates and the value of $j$ varies from 1 to 306 atoms.

Equation (2) can be put in the form to represent the cohesive energy at equilibrium semi-lattice constant $\left(a_{0}\right)$ as below

$$
\begin{aligned}
\Phi\left(a_{0}\right) & =[D / 2(m-1)] \\
\times & {\left[\beta^{m}\left(\alpha a_{0}\right)^{-n} \sum_{l_{1} l_{2} l_{3}} L_{j}^{-n} \mathrm{e}^{-m \alpha a_{0} L_{j}}-m \beta\left(\alpha a_{0}\right)^{n} \sum_{l_{1} l_{2} l_{3}} L_{j}^{n} \mathrm{e}^{-\alpha a_{0} L_{j}}\right], }
\end{aligned}
$$

where

$$
\beta=\exp \left(\alpha r_{0}\right)
$$

The three defining parameters $\left(\alpha, r_{0}\right.$, and $\left.D\right)$ of the potential require for their evaluation the precisely determined input data of equilibrium semi-lattice constant $\left(a_{0}\right)$ and bulk modulus $(B)$ of the metals only (Table I). For evaluating the three parameters $\alpha, r_{0}$ and $D$ of the potential function, the condition [19]

$$
\sum_{l_{1} l_{2} l_{3}}\left[l_{1}^{2} \mathrm{~d} \Phi(r) / \mathrm{d} r^{2}\right]=0
$$

for the equilibrium of the crystal in the absence of external forces was employed and executed (Table II).

\section{TABLE I}

Input data for bcc metals.

\begin{tabular}{l|c|c}
\hline \hline Metal & $\begin{array}{c}\text { Semi-lattice constant } \\
{\left[\times 10^{-10} \mathrm{~m}\right]}\end{array}$ & $\begin{array}{c}\text { Bulk modulus } \\
{\left[\times 10^{11} \mathrm{~N} / \mathrm{m}^{2}\right]}\end{array}$ \\
\hline $\mathrm{V}$ & 1.515 & 1.619 \\
$\mathrm{Nb}$ & 1.650 & 1.702 \\
$\mathrm{Ta}$ & 1.650 & 2.000
\end{tabular}

TABLE II

Computed potential parameters for bcc metals.

\begin{tabular}{l|c|c|c|c|c|c|c}
\hline \hline Metal & $n$ & $m$ & $\alpha a_{0}$ & $\begin{array}{c}\alpha \\
{\left[\times 10^{10} \mathrm{~m}^{-1}\right]}\end{array}$ & $\beta$ & $\begin{array}{c}D \\
{\left[\times 10^{-26} \mathrm{~J}\right]}\end{array}$ & $\begin{array}{c}r_{0} \\
{\left[\times 10^{-9} \mathrm{~m}\right]}\end{array}$ \\
\hline $\mathrm{V}$ & 2 & 1.5 & 2.0989 & 1.385413 & 2186878 & 5.943871 & 1.053692 \\
$\mathrm{Nb}$ & 2 & 1.5 & 2.0194 & 1.223879 & 1594490 & 10.324240 & 1.166950 \\
$\mathrm{Ta}$ & 2 & 1.5 & 2.1719 & 1.316303 & 2923407 & 7.549511 & 1.131066
\end{tabular}

\subsection{Theoretical considerations}

The present calculations are based on the theory and procedure proposed by Milstein [13] in considerable detail for applying the Born stability criteria to the determination of the mechanical stability of cubic crystals in the presence of 
applied forces and deformations. Therefore, only few essential aspects are mentioned here. For [100] uniaxial stress in cubic crystals with central interactions, the necessary and sufficient conditions for a lattice to be in stable equilibrium are

$$
\begin{aligned}
& B_{11}>0, \quad B_{22}>0, \quad B_{12}>0, \quad B_{23}>0, \\
& A A=B_{22}-B_{23}>0, \\
& B B=B_{11}\left(B_{22}+B_{23}\right)-2\left(B_{12}\right)^{2}>0
\end{aligned}
$$

where

$$
B_{i j}=\frac{\partial^{2} E}{\partial a_{i} \partial a_{j}}
$$

and $E$ is the energy per unit cell given by

$$
E=(1 / 2) n^{\prime} \sum_{l_{1} l_{2} l_{3}} \phi(r) .
$$

$i$ and $j$ have their values ranging from 1 to 6 and $n^{\prime}$ is the number of atoms per unit cell. $a_{1}, a_{2}$ and $a_{3}$ are the edges of the unit cell and $a_{4}, a_{5}$ and $a_{6}$ are the angles between half the edges $a_{2}$ and $a_{3}, a_{3}$ and $a_{1}, a_{1}$ and $a_{2}$ respectively. The normal stress acting on a face of the unit cell, when the cell edges are perpendicular to each other is given by

$$
\sigma=\frac{1}{a_{2} a_{3}}\left[\frac{\partial E}{\partial a_{1}}\right] \text {. }
$$

The force $F_{1}$ acting on the crystal lattice in the direction $a_{1}$ is given by i.e. uniaxial (100) expansion or contraction

$$
F_{1}=\left[\frac{\partial E}{\partial a_{1}}\right]
$$

and the force $F_{2}$ acting along $a_{2}$ is

$$
F_{2}=\left[\frac{\partial E}{\partial a_{2}}\right]=0 \text {, }
$$

where for a tetragonal crystal lattice

$$
a_{2}=a_{3} \text { and } a_{4}=a_{5}=a_{6}=\pi / 2 \text {. }
$$
bcc lattice)

The moduli $B_{i j}$ embodied in Eqs. (7) to (9) are given by (with $n^{\prime}=2$ for

$$
\begin{aligned}
& B_{11}=a_{1}^{2} \sum_{l_{1} l_{2} l_{3}} l_{1}^{4} \frac{\partial^{2} \phi}{\left(\partial r^{2}\right)^{2}}+(1 / 2) \sum_{l_{1} l_{2} l_{3}} l_{1}^{2} \frac{\partial \phi}{\partial r^{2}} \\
& B_{22}=a_{2}^{2} \sum_{l_{1} l_{2} l_{3}} l_{2}^{4} \frac{\partial^{2} \phi}{\left(\partial r^{2}\right)^{2}}+(1 / 2) \sum_{l_{1} l_{2} l_{3}} l_{2}^{2} \frac{\partial \phi}{\partial r^{2}} \\
& B_{12}=a_{1} a_{2} \sum_{l_{1} l_{2} l_{3}} l_{1}^{2} l_{2}^{2} \frac{\partial^{2} \phi}{\left(\partial r^{2}\right)^{2}}
\end{aligned}
$$




$$
B_{23}=a_{2} a_{3} \sum_{l_{1} l_{2} l_{3}} l_{2}^{2} l_{3}^{2} \frac{\partial^{2} \phi}{\left(\partial r^{2}\right)^{2}},
$$

where $a_{1}$ and $a_{2}$ stand for semi-lattice constants and for a tetragonal crystal lattice

$$
r=\left[l_{1}^{2} a_{1}^{2}+\left(l_{2}^{2}+l_{3}^{2}\right) a_{2}^{2}\right]^{1 / 2} .
$$

\section{Computations and results}

The behaviour of perfect bcc crystals of $\mathrm{V}, \mathrm{Nb}$ and $\mathrm{Ta}$ are studied for (100) uniaxial tensile and compressive stresses. In order to compute the theoretical strength and stability, the condition of lattice equilibrium $\partial E / \partial a_{1}=$ $\partial E / \partial a_{2}=\partial E / \partial a_{3}=0$ is satisfied at experimentally known equilibrium values of semi-lattice constants $a_{1}=a_{2}=a_{3}=a^{0}$. Under the action of force $F_{1}$ (Eq. (13)), the semi-lattice constant $a_{1}$ is given small increments and decrements to make nucleation effective such that symmetrical changes in $a_{2}$ and $a_{3}$ satisfy $\partial E / \partial a_{2}=\partial E / \partial a_{3}=0$ at each stage of deformation in $a_{1}$. The process of iteration was applied to carry out these computations and continued until one of the stability conditions (Eq. (7) to Eq. (9)) is violated. The value of $F_{1}^{\mathrm{f}} /\left(a_{2}^{\mathrm{f}}\right)^{2}$ at which the instability occurs is the theoretical strength (stress) of the crystal and $\left(a_{1}{ }^{f}-a_{1}^{0}\right) / a_{1}^{0}$ is the theoretical maximal strain, where $f$ refers to the final stage at which instability occurs.

For a tensile force, the edge $a_{1}$ will elongate and the edges $a_{2}$ and $a_{3}$ will contract such that $a_{2}=a_{3}$ and $a_{4}=a_{5}=a_{6}=\pi / 2$. Then, the deformed crystal will possess "tetragonal symmetry" and bcc crystal lattice transforms into body centered tetragonal (bct) phase, as a result of nucleation of the crystal lattice. Similarly, for a compressive force, the reverse will be the effect of nucleation. At each stage of deformation, the numerical values of the moduli $B_{i j}$, applied stress and internal energy are calculated.

\section{Conclusions}

A close survey of the literature reveals that even today, there exist not enough experimental data on a wide variety of materials, involving different modes of failure, nor there has been a sufficient number of theoretical calculations of stability, in different modes of applied stress, to make detailed quantitative comparison between the theoretical and experimental behaviour in particular failure modes. Nevertheless, it is still satisfying to note that a variety of metallic whiskers have exhibited maximum stresses in the range of about $0.17 \times 10^{10} \mathrm{~N} / \mathrm{m}^{2}$ (for $\mathrm{Ag}$ ) to about $1.31 \times 10^{10} \mathrm{~N} / \mathrm{m}^{2}$ (for $\mathrm{Fe}$ ) with a corresponding strains estimated to be about $3 \%$ to $5 \%$ [20-22].

The effect of nucleation i.e. instability in tension for the bcc lattice results from the violation of the condition defined by Eq. (9). Evidently this relation (Eq. (9)) becomes violated when a further increase in semi-lattice constant $a_{1}$ leads to a decrease in the equilibrium stress i.e. when the lattice cannot accommodate a further increase in applied stress.

The effect of nucleation i.e. instability in compression for the bcc lattice results from the violation of the condition defined by Eq. (8). The violation of this 
condition corresponds to the mode of failure by which the crystal can lower its total energy by undergoing spontaneously the following lattice deformation.

The variation of internal energy defined by Eq. (11) with semi-lattice constant $a_{1}$ for the bcc metals under study shows two distinct minima, one for bcc phase (where stress $\sigma_{1}$ becomes negative) and the other for bct phase (where stress $\sigma_{1}=0$ ). It is to be noted that the internal energy minimum (IEM) of the bct phase is considerably lower than that of the bcc phase. The unstressed $\left(\sigma_{1}=0\right)$ bct phase possesses the semi-lattice constant $a_{1}=b_{1}^{0}$ and $a_{2}=b_{2}^{0}$ corresponding to the minimum of internal energy. The values of semi-lattice constants $a_{1}, a_{2}$ and the corresponding values of the energy minima for the bcc and bct phases of the metals studied are given in Table III.

TABLE III

Internal energy minima (IEM) and stress.

\begin{tabular}{l|c|c|c|c}
\hline \hline \multicolumn{5}{c}{ (a) bcc phase } \\
\hline Metal & $a_{1}[\AA]$ & $a_{2}[\AA]$ & $\begin{array}{c}\text { Minima position } \\
{\left[10^{-19} \mathrm{~J} / \text { unit cell }\right]}\end{array}$ & $\begin{array}{c}\text { Siress } \\
{\left[10^{9} \mathrm{~N} / \mathrm{m}^{2}\right]}\end{array}$ \\
\hline $\mathrm{V}$ & 1.59075 & 1.478295 & -16.991 & -0.0468 \\
$\mathrm{Nb}$ & 1.76550 & 1.594449 & -24.224 & -0.5077 \\
$\mathrm{Ta}$ & 1.73250 & 1.609795 & -25.920 & -0.3241 \\
\hline \multicolumn{5}{c}{ (b) bct phase } \\
\hline Metal & $a_{1}=b_{1}^{0}[\AA]$ & $a_{2}=b_{2}^{0}[\AA]$ & Minima position & Stress \\
& & & {$\left[10^{-19} \mathrm{~J} /\right.$ unit cell $]$} & {$\left[10^{9} \mathrm{~N} / \mathrm{m}^{2}\right]$} \\
\hline $\mathrm{V}$ & 1.931625 & 1.338190 & -17.079 & 0.0 \\
$\mathrm{Nb}$ & 2.095500 & 1.459299 & -24.319 & 0.0 \\
$\mathrm{Ta}$ & 2.163750 & 1.456480 & -26.069 & 0.0
\end{tabular}

The bct lattice fails in tension due to increased nucleation by violating the condition $B_{23}>0$. When this condition is violated, the lattice can lower its energy by undergoing a spontaneous deformation wherein the angle $a_{4}$ (the angle between $a_{2}$ and $a_{3}$ ) deviates from $90^{\circ}$.

The bct lattice fails in compression due to increased nucleation by violating the condition defined by Eq. (9). The violation of this condition results when the lattice cannot support an additional compressive load i.e. when a decrease in semi-lattice constant $a_{1}$ results in an increase rather than a decrease in stress $\sigma_{1}$.

It is to be noted that the range of stability of the bet phase is considerably greater than that of the bcc phase.

We now discuss below our findings for bcc metallic crystals in the framework of extended generalised exponential potential, concerning the moduli $B_{i j}$, applied stress and internal energy. 


\subsection{Vanadium}

Figures 1 and 2 show the variation of $B_{i j}$ as a function of semi-lattice constant $a_{1}$ for V. Figure 3 shows the variation of $A A=B_{22}-B_{23}$ and $B B=$ $B_{11}\left(B_{22}+B_{23}\right)-2 B_{12}^{2}$ as a function of semi-lattice constant $a_{1}$ for V. Figure 4 shows the variation of applied stress and internal energy of $\mathrm{V}$ as a function of semi-lattice constant $a_{1}$. It follows from Fig. 3 and Fig. 4 that bcc lattice of V becomes unstable at a theoretical tensile stress of $0.4698 \times 10^{9} \mathrm{~N} / \mathrm{m}^{2}$ and a corresponding strain of $1.75 \%$ when $B B<0$ and in compression, the bcc lattice of $\mathrm{V}$ becomes unstable at an applied stress of $-0.7466 \times 10^{9} \mathrm{~N} / \mathrm{m}^{2}$ with a strain of $1.65 \%$ when $A A<0$ for $n=2, m=1.5$ and $\alpha a_{0}=2.0989$. Thus the range of stability of $\mathrm{V}$ is from $a_{1}=1.541513 \AA, a_{2}=1.502205 \AA$ to $a_{1}=1.490003 \AA$, $a_{2}=1.527006 \AA$.

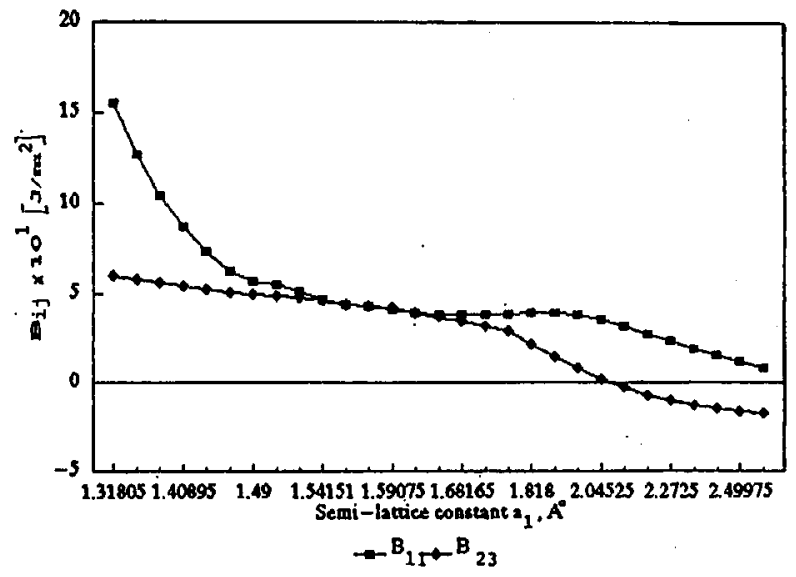

Fig. 1. Variation of $B_{11}$ and $B_{23}$ as a function of semi-lattice constant for V.

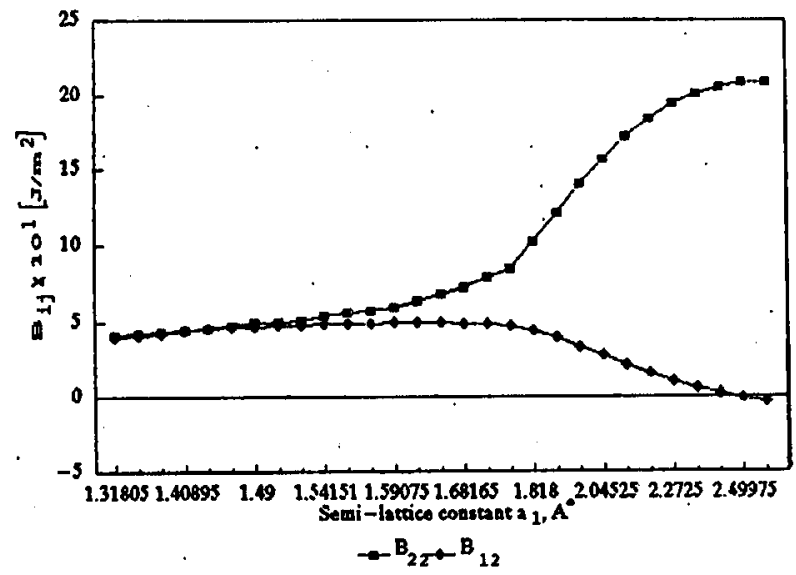

Fig. 2. Variation of $B_{22}$ and $B_{12}$ as a function of semi-lattice constant for V. 


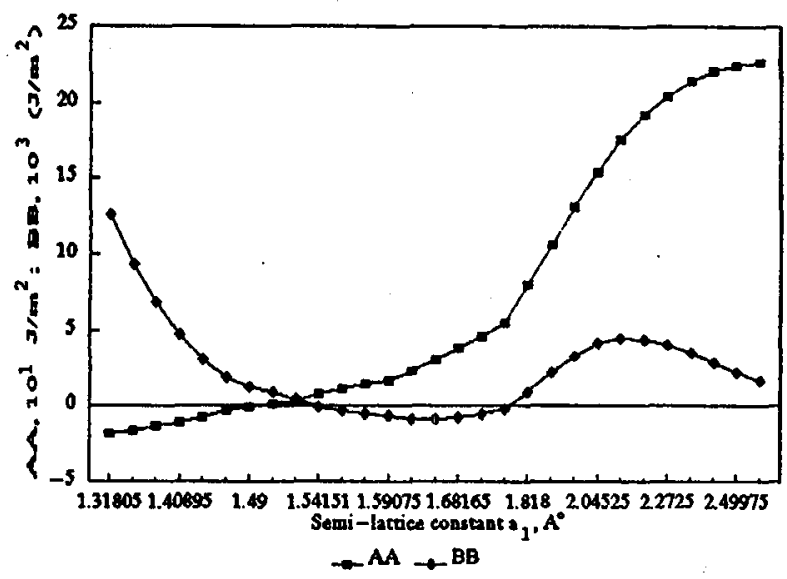

Fig. 3. Variation of $A A$ and $B B$ as a function of semi-lattice constant for V.

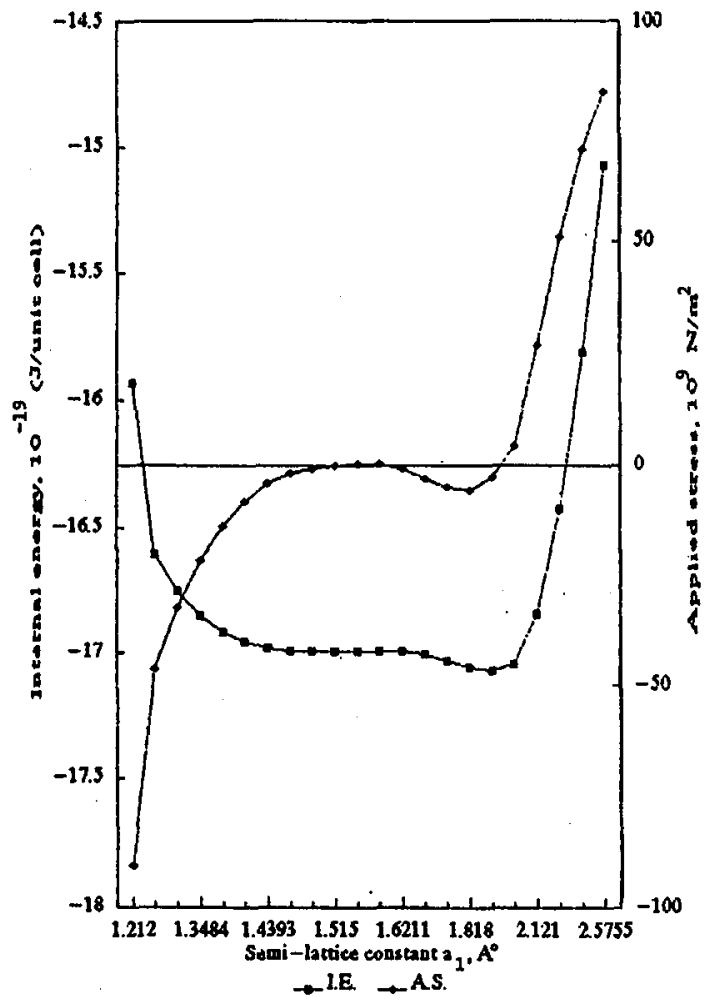

Fig. 4. Variation of internal energy and applied stress as a function of semi-lattice constant for $\mathrm{V}$. 
It follows from Fig. 4 that the theoretical strength of bct phase of $\mathrm{V}$ is $2.065 \times 10^{10} \mathrm{~N} / \mathrm{m}^{2}$ in tension when $B_{23}<0$ and $-0.56 \times 10^{10} \mathrm{~N} / \mathrm{m}^{2}$ in compression when Eq. (9) is violated; the corresponding strains being $7.84 \%$ in tension and $-5.88 \%$ in compression. The bct phase of $\mathrm{V}$ is stable within the range of semi-lattice constants $a_{1}=1.818 \AA, a_{2}=1.37624 \AA$ to $a_{1}=2.083125 \AA, a_{2}=1.30338 \AA$.

\subsection{Niobium}

Figures 5 and 6 show the variation of $B_{i j}$ as a function of semi-lattice constant $a_{1}$ for Nb. Figure 7 shows the variation of $A A=B_{22}-B_{23}$ and $B B=B_{11}\left(B_{22}+B_{23}\right)-2 B_{12}^{2}$ as a function of semi-lattice constant $a_{1}$ for $\mathrm{Nb}$. Figure 8 shows the variation of applied stress and internal energy of $\mathrm{Nb}$ as a function of semi-lattice constant $a_{1}$. Figures 7 and 8 show that bcc lattice of

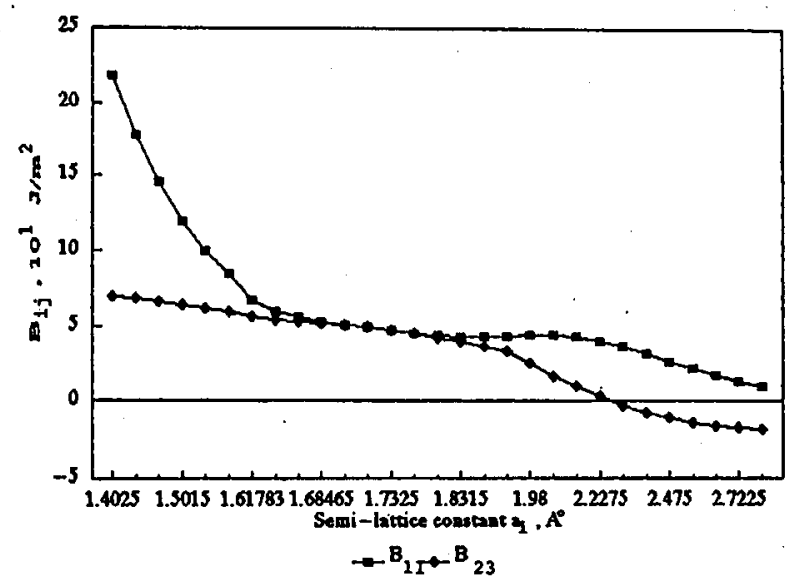

Fig. 5. Variation of $B_{11}$ and $B_{23}$ a function of semi-lattice constant for $\mathrm{Nb}$.

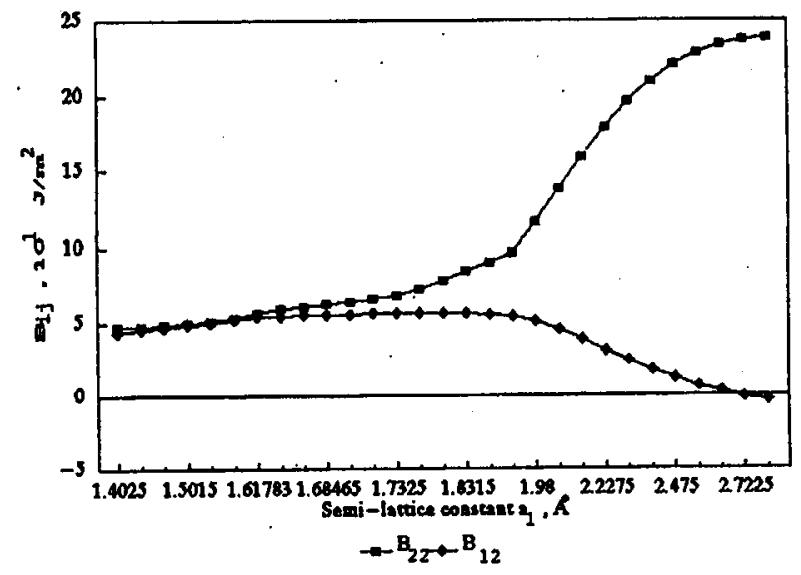

Fig. 6. Variation of $B_{22}$ and $B_{12}$ as a function of semi-lattice constant for $\mathrm{Nb}$. 


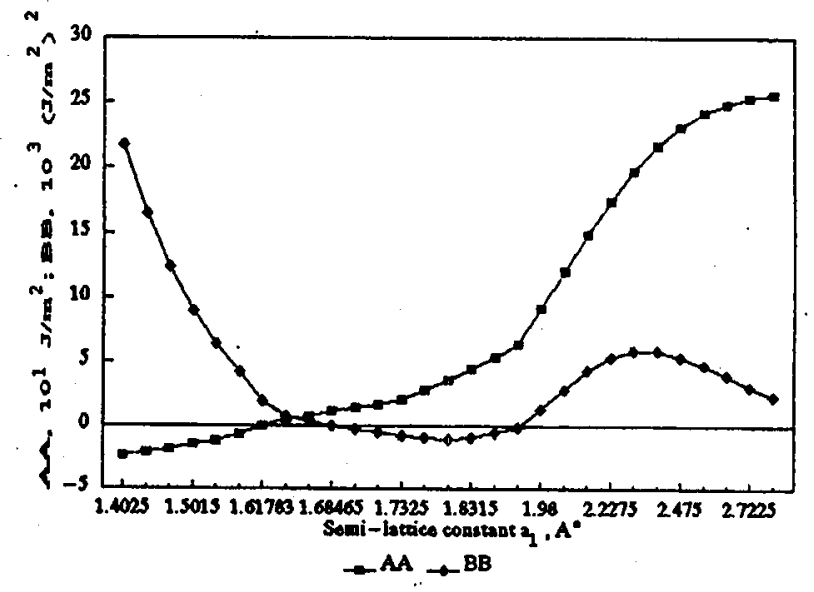

Fig. 7. Variation of $A A$ and $B B$ as a function of semi-lattice constant for $\mathrm{Nb}$.

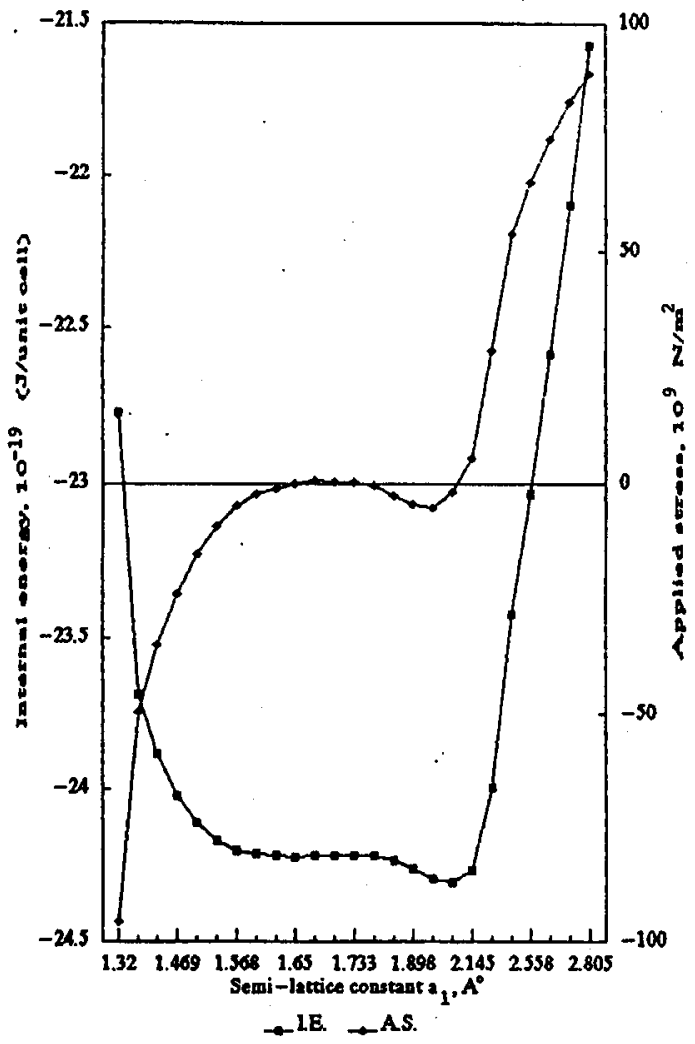

Fig. 8. Variation of internal energy and applied stress as a function of semi-lattice constant for $\mathrm{Nb}$. 
$\mathrm{Nb}$ becomes unstable at a theoretical tensile stress of $0.6875 \times 10^{9} \mathrm{~N} / \mathrm{m}^{2}$ and a corresponding strain of $2.1 \%$ when $B B<0$ and in compression, the bcc lattice of $\mathrm{Nb}$ becomes unstable at an applied stress of $-1.1017 \times 10^{9} \mathrm{~N} / \mathrm{m}^{2}$ with a strain of $1.95 \%$ when $A A<0$ for $n=2, m=1.5$ and $\alpha a_{0}=2.0194$. Thus the range of stability of $\mathrm{Nb}$ is from $a_{1}=1.68465 \AA, a_{2}=1.633385 \AA$ to $a_{1}=1.617825 \AA$, $a_{2}=1.665341 \AA$.

Figure 8 shows that the theoretical strength of bct phase of $\mathrm{Nb}$ is $2.2 \times 10^{10} \mathrm{~N} / \mathrm{m}^{2}$ in tension when $B_{23}<0$ and $-0.499 \times 10^{10} \mathrm{~N} / \mathrm{m}^{2}$ in compression when Eq. (9) is violated; the corresponding strains $8.27 \%$ in tension and $-5.51 \%$ in compression. The bct phase of $\mathrm{Nb}$ is stable within the range of semi-lattice constants $a_{1}=1.98 \AA, a_{2}=1.499948 \AA$ to $a_{1}=2.26875 \AA, a_{2}=1.420672 \AA$.

\subsection{Tantalum}

Figures 9 and 10 exhibit the variation of $B_{i j}$ as a function of semi-lattice constant $a_{1}$ for bcc Ta. Figure 11 shows the variation of $A A=B_{22}-B_{23}$ and $B B=B_{11}\left(B_{22}+B_{23}\right)-2 B_{12}^{2}$ as a function of semi-lattice constant $a_{1}$ for bcc Ta. Figure 12 shows the variation of applied stress and internal energy of bcc Ta as a function of semi-lattice constant $a_{1}$. It follows from Fig. 11 and Fig. 12 that bcc lattice of Ta becomes unstable at a theoretical tensile stress of $0.4339 \times 10^{9} \mathrm{~N} / \mathrm{m}^{2}$ and a corresponding strain of $1.47 \%$ when $B B<0$ and in compression, the bcc lattice of Ta becomes unstable at an applied stress of $-0.6599 \times 10^{9} \mathrm{~N} / \mathrm{m}^{2}$ with a strain of $1.38 \%$ when $A A<0$ for $n=2, m=1.5$ and $\alpha a_{0}=2.1719$. Thus the range of stability of bcc Ta is from $a_{1}=1.674255 \AA, a_{2}=1.638234 \AA$ to $a_{1}=1.62723 \AA, a_{2}=1.661007 \AA$.

It follows from Fig. 12 that the theoretical strength of bct phase of $\mathrm{Ta}$ is $2.35 \times 10^{10} \mathrm{~N} / \mathrm{m}^{2}$ in tension when $B_{23}<0$ and $-0.785 \times 10^{10} \mathrm{~N} / \mathrm{m}^{2}$ in compression when Eq. (9) is violated; the corresponding strains $7.45 \%$ in tension and $-5.88 \%$

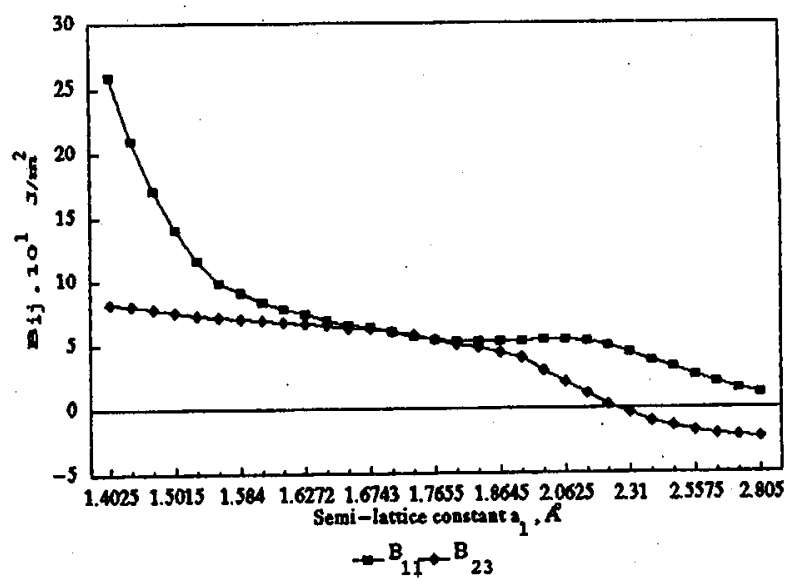

Fig. 9. Variation of $B_{11}$ and $B_{23}$ as a function of semi-lattice constant for Ta. 


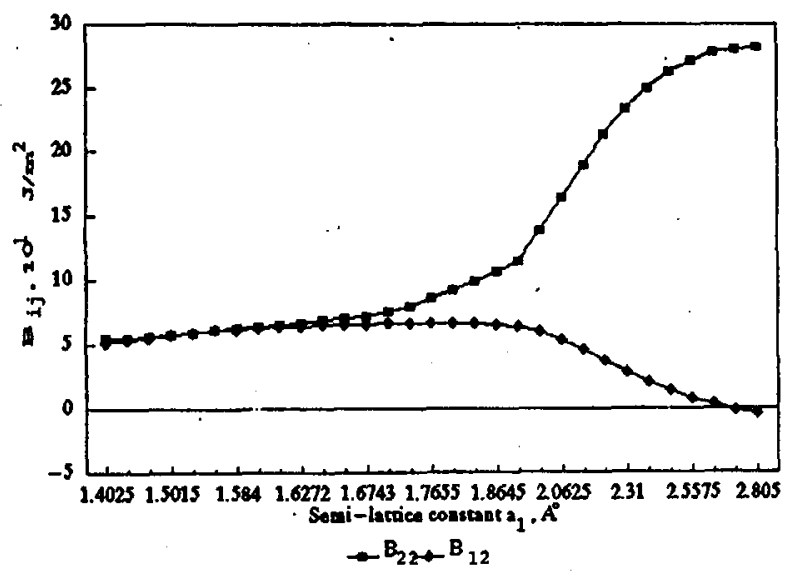

Fig. 10. Variation of $B_{22}$ and $B_{12}$ as a function of semi-lattice constant for Ta.

TABLE IV

Computed values of $C_{11}$ and $C_{12}$ for bcc phase in $10^{11} \mathrm{~N} / \mathrm{m}^{2}$.

\begin{tabular}{l|l|l|l}
\hline \hline Metal & \multicolumn{1}{|c|}{$C_{11}$} & \multicolumn{1}{|c|}{$C_{12}$} & \multicolumn{1}{c}{ Remark } \\
\hline $\mathrm{V}$ & 1.6974 & 1.5769 & At equilibrium \\
& 1.5221 & 1.5713 & At failure in tension \\
& 1.9157 & 1.5776 & At failure in compression \\
Expt. & 2.280 & 1.187 & {$[23]$} \\
& 1.96 & 1.33 & {$[24]$} \\
\hline $\mathrm{Nb}$ & 1.7994 & 1.6508 & At equilibrium \\
& 1.5797 & 1.6419 & At failure in tension \\
& 2.0815 & 1.6513 & At failure in compression \\
Expt. & 2.46 & 1.34 & {$[24]$} \\
\hline Ta & 2.0820 & 1.9553 & At equilibrium \\
& 1.8994 & 1.9503 & At failure in tension \\
& 2.2997 & 1.9561 & At failure in compression \\
Expt. & 2.609 & 1.574 & [23]
\end{tabular}

in compression. The bct phase of $\mathrm{Ta}$ is stable within the range of semi-lattice constants $a_{1}=1.98 \AA, a_{2}=1.498008 \AA$ to $a_{1}=2.2605 \AA, a_{2}=1.419068 \AA$.

Finally, the second-order elastic constants (Table IV) $C_{11}$ and $C_{12}$ are calculated as per Milstein [13] at the stress-free equilibrium values of semi-lattice constant and at failure in tension and compression values of semi-lattice constant. It is observed that in general computed values of elastic constants $\left(C_{11}\right.$ and $\left.C_{12}\right)$ decreases in tension and increases in compression, due to the effect of nucleation. 


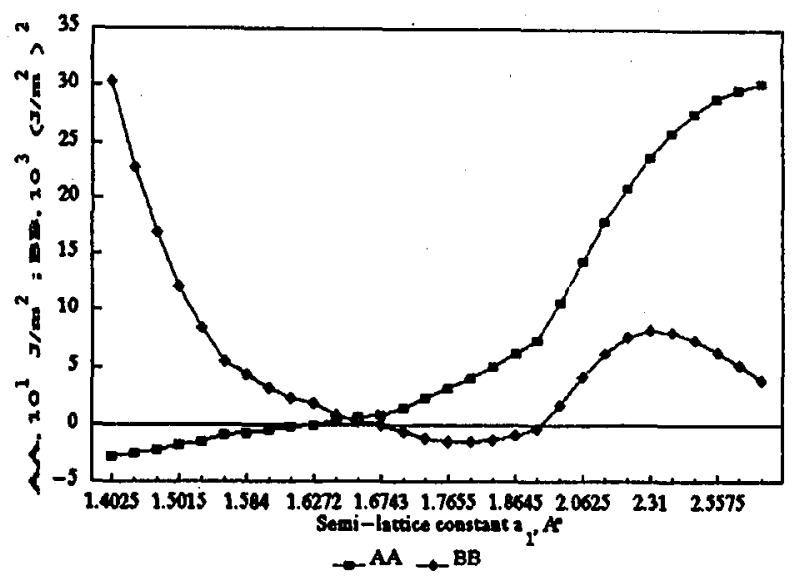

Fig. 11. Variation of $A A$ and $B B$ as a function of semi-lattice constant for Ta.

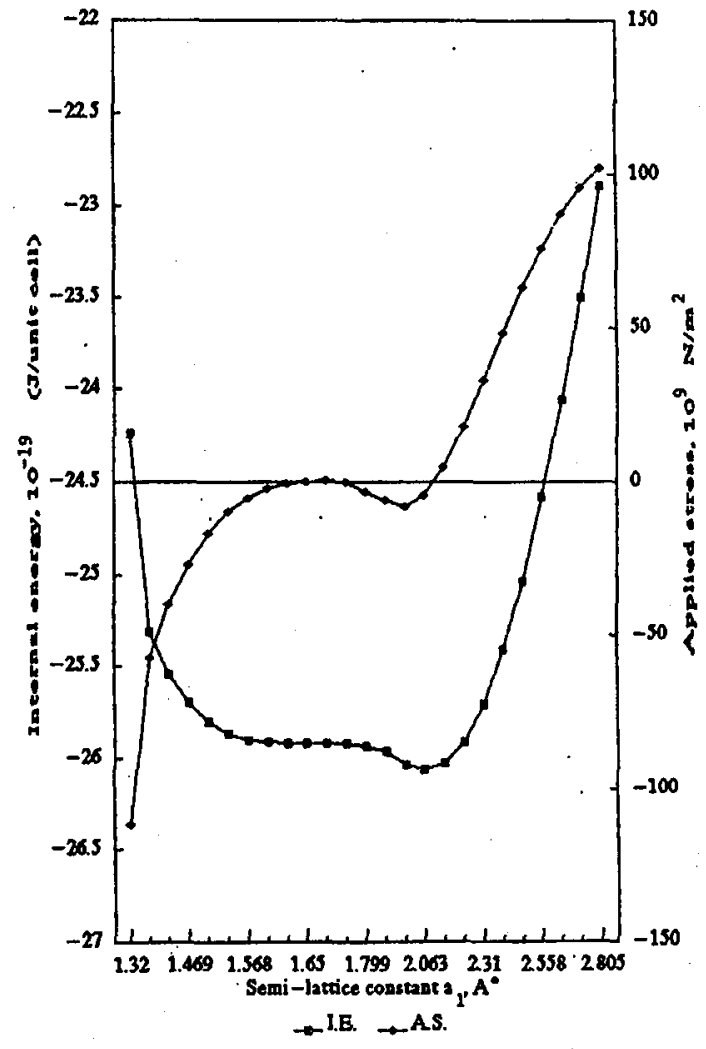

Fig. 12. Variation of internal energy and applied stress as a function of semi-lattice constant for Ta. 


\section{Acknowledgments}

Two of us (A.V. and M.L.V.) are thankful to Mr. N.K. Khurana of Universal Computer Training Point (UCTP) for providing computation facility and to Dr. G. Singh, R.B.S. College, Agra, for many useful discussions.

\section{References}

[1] M. Born, Proc. Camb. Philos. Soc. 36, 160 (1940).

[2] I.H. Khlyustkov, A.T. Buzdin, Adv. Phys. 36, 271 (1987); J.G. Bednorz, K.A. Mueller, Z. Phys. B 64, 189 (1986); Z.Z. Sheng, A.M. Herman, Nature 332, 138 (1988).

[3] M. Born, R. Furth, Proc. Camb. Philos. Soc. 36, 454 (1940).

[4] A. Kelly, Strong Solids, Clarendon Press, Oxford 1966.

[5] W.R. Tyson, Philos. Mag. 14, 925 (1966).

[6] N.H. Macmillan, J. Mater. Sci. 7, 239 (1972).

[7] A. Kelly, W.R. Tyson, A.A. Cottrell, Philos. Mag. 15, 567 (1967).

[8] P.C. Ghlen, A.R. Rosenfield, G.T. Hahn, J. Appl. Phys. 39, 5246 (1968).

[9] Z.S. Basinski, M.S. Duesbery, R. Taylor, Philos. Mag. 21, 1201 (1970).

[10] M.F. Ashbay, S.H. Gelles, L.E. Tanner, Philos. Mag. 19, 757 (1969).

[11] L.M. Brown, G.R. Woolhouse, Philos. Mag. 21, 329 (1970).

[12] R.D. Misra, Proc. Camb. Philos. Soc. 36, 173 (1940).

[13] F. Milstein, Phys. Rev. B 2, 512 (1971); Phys. Rev. B 3, 1130 (1971).

[14] F. Milstein, Phys. Rev. B 10, 3635 (1974); J. Appl. Phys. 44, 3833 (1973).

[15] A.K. Mitra, P.K. Sengupta, J. Phys. F 13, 2221 (1983).

[16] G. Singh, R.P.S. Rathore, Indian J. Pure Appl. Phys. 24, 303 (1986).

[17] G. Singh, Indian J. Pure Appl. Phys. 28, 122 (1990); Phys. Status Solidi B 161, 145 (1990); Phys. Status Solidi B 164, 401 (1991).

[18] A. Verma, M.L. Verma, R.P.S. Rathore, Acta Phys. Pol. A 90, 547 (1996);

M.L. Verma, A. Verma, R.P.S. Rathore, Indian J. Phys. A 70, 603 (1996).

[19] F. Milstein, J. Appl. Phys. 44, 3825 (1973).

[20] S.S. Brenner, J. Appl. Phys. 27, 1484 (1956).

[21] S.S. Brenner, Science 128, 569 (1958).

[22] J.C. Crump, J.W. Mitchell, J. Appl. Phys. 41, 717 (1970).

[23] C. Kittel, Introduction to Solid State Physics, 3rd ed., Wiley, New:York 1968, p. 122.

[24] I.D. Bolef, J. Appl. Phys. 32, 100 (1961). 